\title{
ALIMENTAÇÃO E SAÚDE: SENTIDOS ATRIBUÍDOS POR ADOLESCENTES
}

\author{
Food and health: meanings attributed by adolescents \\ Alimentación y salud: sentidos atribuidos por adolescentes
}

\section{RESUMO}

Objetivos: identificar o saber de adolescentes sobre uma alimentação saudável, analisar os alimentos que compõem suas refeições, discutir este consumo à luz de uma alimentação saudável e programar ações de educação em saúde sobre o tema. Método: Pesquisa convergente-assistencial, com entrevista individual e aplicação de um roteiro com questões fechadas e abertas. Participaram 14 adolescentes, entre 14 e 17 anos, de um colégio público. Resultados: apesar de os adolescentes saberem sobre os hábitos saudáveis de alimentação, a maioria declarou não os seguir. A cultura de alimentação com comidas de preparação rápida marca seus hábitos, podendo trazer consequências à sua saúde. Conclusão: intervenções são necessárias para a melhoria dos hábitos alimentares dos adolescentes, para que eles perdurem na fase adulta. Como parte da pesquisa convergente-assistencial, a educação em saúde, baseada no diálogo, investiu no esclarecimento dos adolescentes sobre o tema.

Palavras-chave: Adolescente. Alimentação. Cuidados de enfermagem. Educação em saúde.

\begin{abstract}
The purposes of this study are to identify the teenagers' knowledge about healthy feeding generally consumed by them, as well as discuss their consumption compared to the healthy feeding and to program health education actions about the subject. It is a convergent-assistance research, with individual interviews and application of a script with open and closed questions. The sample was comprised to 14 teenagers, with ages ranging from 14 to 17 years from a public school of the city. The results show us that the teenagers know about the healthy feeding habits, but most of them do not make use of these habits. The fast food culture is present in their habits that may have consequences for their health. It was concluded that the interventions are necessary to improve the teenagers healthy feeding, in order to last during the adulthood. As part of the convergent-assistance research, the intervention was done through dialogue and clarification of the teenagers questions about the subject.
\end{abstract}

Keywords: Adolescent. Feeding. Nursing care. Health education.

\section{Resumen}

Objetivos: identificar el saber de adolescentes sobre una alimentación saludable, analizar los alimentos que componen sus comidas, discutir este consumo a la luz de una alimentación saludable y programar acciones de educación en salud sobre el tema. Método: Investigación convergente-asistencial, con entrevista individual y aplicación de una encuesta con preguntas cerradas y abiertas. Participaron 14 adolescentes entre 14 y 17 años, de un colegio público. Resultados: además de los adolescentes conocieren los hábitos saludables de alimentación, la mayoría declaró no los seguir. La cultura de alimentación con comidas de preparación rápida marca sus hábitos, lo que puede generar consecuencias a la salud. Conclusión: las intervenciones son necesarias para la mejoría de los hábitos alimentares de los adolescentes, para que los mismos perduren en la fase adulta. Como parte de la investigación convergente-asistencial, la educación en salud, basada en el diálogo, invirtió en la aclaración de los adolescentes sobre el tema.

Palabras claves: Adolescente. Alimentación. Atención de enfermería. Educación en salud.

\footnotetext{
${ }^{1}$ Aluna do Curso de Graduação em Enfermagem da Escola de Enfermagem Anna Nery (EEAN), da Universidade Federal do Rio de Janeiro (UFRJ). Membro do Núcleo de Pesquisa de Fundamentos do Cuidado de Enfermagem (Nuclearte). Bolsista de Iniciação Científica pela Fundação de Apoio a Pesquisa do Estado do Rio de Janeiro (FAPERJ). Rio de Janeiro - RJ. Brasil. E-mail:julyanagall@hotmail.com; ${ }^{2}$ Doutora em Enfermagem. Professora Adjunta do Departamento de Enfermagem Fundamental da EEAN/UFRJ. Membro do Nuclearte. Rio de Janeiro - RJ. Brasil. E-mail: mlot@uol.com.br; ${ }^{3}$ Doutora em Enfermagem. Professora Titular do Departamento de Enfermagem Fundamental da EEAN/UFRJ. Pesquisadora do CNPq. Membro da Diretoria do Nuclearte. Rio de Janeiro - RJ. Brasil. E-mail: marciadeaf@ibest.com.br
} 


\section{INTRODUÇÃO}

A adolescência é uma etapa evolutiva na qual culmina todo o processo maturativo biopsicossocial do indivíduo. Segundo a Organização Mundial da Saúde, a adolescência vai dos 10 aos 20 anos incompletos. Já no Estatuto da Criança e do Adolescente-ECA (Lein. 8069, de 13 de julho de 1990. Brasília: Senado Federal; 1990), ela inicia aos 12 e termina aos 18 anos. Neste período ocorrem intensas modificações corporais, tendo o estado nutricional do adolescente importância fundamental no seu desenvolvimento ${ }^{1}$.

A nutrição relaciona-se diretamente com as modificações corporais dos adolescentes, principalmente aos padrões alimentares adotados por essa faixa da população. As preferências alimentares de cada um são formuladas desde a infância através de sensações e experimentos que são oferecidos a criança através do tato, sabor e odor. Tais preferências vão construindo sensações e significados de representações físicas, sociais, psicológicas e culturais e que irão moldar o compor tamento alimentar de toda uma vida. ${ }^{2}$

A alimentação e a nutrição adequadas constituem requisitos essenciais à confirmação do completo potencial de crescimento e desenvolvimento com qualidade de vida, bem como à prevenção de doenças carenciais a obesidade e comorbidades associadas, além de constituir-se em direito humano indispensável à construção de cidadania. Assim identifica-se a relação da educação nutricional com a promoção da saúde enfatizados por uma das diretrizes da Política Nacional de Alimentação e Nutrição do Ministério da Saúde que é a promoção de práticas alimentares e estilos de vida saudáveis. ${ }^{3}$

A educação nutricional contribui para que 0 adolescente compreenda suas práticas e comportamentos, conhecimentos ou aptidões, proporcionando condições para a tomada de decisões e resolução de problemas que possam se apresentar ao adolescente, mediante os fatos percebidos. ${ }^{4}$

Sabe-se que as práticas alimentares inadequadas são cada vez mais frequentes em adolescentes, sendo o seu padrão alimentar caracterizado pelo consumo excessivo de refrigerantes, açúcares e comidas de preparação rápida e reduzida ingestão de hortaliças e frutas. Também se torna preocupante a adoção de dietas monótonas ou novos modismos alimentares, como, por exemplo, a exclusão de algumas refeições diárias. ${ }^{5}$

Neste contexto, a educação em saúde tem papel fundamental na melhoria dos hábitos alimentares dos adolescentes, tendo os profissionais de enfermagem a função de orientar e esclarecer possíveis dúvidas, promovendo ações que estimulem o autocuidado e a adoção de hábitos saudáveis, valorizando 0 adolescente como sujeito do cuidado tanto no ambiente escolar quanto junto à família. Essa atenção é ressaltada uma vez que a adolescência é vista como fenômeno único e diverso, pois as necessidades dos adolescentes dependem de seu meio social, cultural, econômico e psicológico. ${ }^{6}$
A problemática abordada nesse estudo refere-se à qualidade das informações dos adolescentes sobre uma alimentação saudável no que diz respeito aos seus hábitos diários e a influência do seu meio social nos mesmos. Assim, questiona-se: 0 que sabem os adolescentes sobre alimentação saudável? Que medidas podem ser implementadas para melhor orientar os hábitos alimentares deste grupo etário?

Os objetivos do estudo são identificar o que sabem os adolescentes sobre uma alimentação dita saudável, analisar quais alimentos compõem suas refeições diárias e discutir este consumo em relação ao que se preconiza para uma alimentação saudável e balanceada, e, a partir daí, programar ações de intervenção educativa que visem melhorar a qualidade de vida do grupo estudado, no que tange à alimentação. Ressalta-se que, à luz do método empregado, algumas ações de cunho interventivo-educativo foram implementadas no decorrer do próprio processo da pesquisa, sendo este, também, um objetivo do estudo.

A importância desta pesquisa reside no fato de que a fase da adolescência ocupa lugar de destaque na definição de costumes e hábitos de toda uma vida. Revisando-se a literatura sobre o tema relacionado ao conhecimento sobre alimentação saudável em adolescentes, observou-se um número reduzido de estudos que colocam o adolescente como protagonista desta questão, enfatizando a opinião deles sobre 0 tema e 0 aprimoramento de estratégias educativas que busquem melhorias na alimentação desse grupo. Ao contrário, o maior quantitativo de trabalhos ampara-se em dados epidemiológicos, estatísticos e na prática alimentar dos adolescentes no que se diz respeito ao que é correto ou não no hábito de se alimentar e no estado nutricional dessa população. ${ }^{7-9}$

A relevância do presente estudo baseia-se principalmente em um dos principais fundamentos do Programa de Saúde do Adolescente (PROSAD/MS), que tem em vista a educação em saúde como melhor forma de prevenção de agravos ${ }^{10} \mathrm{~A}$ educação em saúde gera melhoria nos hábitos alimentares e, assim, evita transtornos alimentares que têm incidência aumentada em adolescentes devido à intensa valorização do corpo e da magreza. ${ }^{11}$

Neste ínterim, ressalta-se que, para que sejam implementadas ações efetivas voltadas à melhoria dos hábitos alimentares dessa população, é necessário identificar o que os adolescentes consideram que seja se alimentar bem, quais alimentos integram seus hábitos cotidianos e o que consideram ser os alimentos básicos para uma vida saudável.

\section{METODOLOGIA}

0 presente estudo é de natureza qualitativa, utilizando-se do método de pesquisa convergente-assistencial, que se preocupa em aliar a prática da pesquisa científica à intervenção em saúde, relacionando o contexto social o qual os sujeitos se inserem com as práticas de saúde a serem realizadas. 0 objetivo é a melhora ou a realização de mudanças que sejam 
benéficas para o estilo de vida do indivíduo e, consequentemente, para a sua saúde. ${ }^{12}$

Os sujeitos foram adolescentes escolares, cuja captação foi realizada em um colégio da rede municipal da cidade de Petrópolis, estado do Rio de Janeiro, após a autorização da sua direção mediante assinatura de um termo específico.

A pesquisa foi realizada em fevereiro de 2010 , e as entrevistas foram feitas no próprio colégio, em dias e horários previamente agendados com a direção e com os adolescentes. Para este estudo, a direção do colégio disponibilizou horário para a participação de uma turma do nono ano que concentrava adolescentes com idade entre 14 e 17 anos. Na primeira abordagem, realizada em sala de aula, foram dados esclarecimentos sobre 0 estudo, e, após esta fase, 18 adolescentes se propuseram a participar. A estes Ihes foram entregues o Termo de Consentimento Livre e Esclarecido e a autorização a ser assinada pelos responsáveis.

Dos 18 adolescentes que receberam a documentação, 14 ( 4 do sexo masculino e 10 do sexo feminino) retornaram com os termos devidamente assinados, sendo este o total de sujeitos pesquisados.

A produção dos dados foi orientada a partir de um questionário misto, com perguntas aber tas e fechadas para responder às questões da pesquisa. A aplicação do instrumento foi feita de forma individual e contou com duas etapas: na primeira, o instrumento foi entregue aos adolescentes para que eles pudessem responder às perguntas fechadas, marcando as opções de respostas no próprio instrumento. As questões do instrumento respondidas nesta etapa eram fechadas e visaram acessar os hábitos cotidianos dos adolescentes no que tange à sua alimentação com vistas a conhecer os alimentos que consomem, os que gostam e que tipos de alimentos consideram saudáveis. Na segunda etapa, as questões abertas exploraram os saberes, em termos de opiniões, definições e representações, sobre o que para eles seja uma alimentação saudável e as relações dos alimentos com a saúde. Esta etapa foi desenvolvida com a aplicação da técnica de entrevista, na qual o pesquisador estabeleceu o diálogo com 0 adolescente dirigindo-lhe as perguntas, sendo todo este diálogo registrado por meio eletrônico.

Destaca-se que esta pesquisa se vincula ao projeto integrado intitulado "Saúde do adolescente: implicações para o cuidado de enfermagem", aprovado pelo Comitê de Ética em Pesquisa da Escola de Enfermagem Anna Nery (EEAN) e Hospital Escola São Francisco de Assis (HESFA) da Universidade Federal do Rio de Janeiro (UFRJ), protocolo número 077/07. Portanto, seguiu-se o preconizado pela Resolução 196/96 do Conselho Nacional de Saúde que trata de pesquisas com seres humanos.

Os dados coletados sofreram dois tipos de análise: às respostas às questões fechadas foram aplicadas regras de contagem simples com o intuito de se ter uma abordagem mais geral para exploração acerca dos alimentos mais e menos consumidos pelos adolescentes; às respostas discursivas foram aplicadas técnicas de análise de conteúdo temático visando apreender as informações de cunho qualitativo que dessem uma visão sobre a opinião dos adolescentes acerca do tema central da pesquisa. As respostas discursivas foram transcritas na íntegra, mantendo-se a fidedignidade do relato dos sujeitos.

Os dados foram organizados em dois blocos: no primeiro, apresentam-se os resultados objetivos, oriundos da primeira parte do instrumento; no segundo, apresentam-se os subjetivos, oriundos da classificação do conteúdo das respostas às questões abertas. Após esta apresentação, a discussão é feita articulando-se os resultados dos dois blocos.

Cabe ressaltar que foi mantido o anonimato dos sujeitos por meio da identificação alfanumérica, na qual a letra $M$ indica o sexo masculino e a F, o feminino, com numeração consecutiva de 1 a 10 para as meninas e de 1 a 4 para os meninos.

\section{RESULTADOS}

\section{Os hábitos alimentares dos adolescentes}

No que concerne aos hábitos cotidianos dos adolescentes no que tange à sua alimentação, identificou-se que o desjejum foi a refeição majoritária, uma vez que todos os adolescentes referiram fazê-la diariamente (14). 0 almoço, lanche e jantar foram citados por 11 adolescentes. A ceia, refeição mais incomum, não teve grande incidência nas respostas (5).

Observa-se que, ainda que o colégio ofereça o almoço para os alunos, nem todos fazem esta refeição, já que três adolescentes informaram não realizá-la cotidianamente.

Sobre os tipos de alimentos ingeridos no almoço ejantar, identificou-se que estas refeições são compostas pela combinação típica da cultura brasileira, em especial do Rio de Janeiro, com arroz (14), feijão (13) e carne vermelha ou de ave (10). 0 macarrão também foi citado por 10 adolescentes. Os acompanhamentos comumente ingeridos são: batata, em vários tipos de preparação, sendo a mais incidente a cozida (9), seguida da frita e em purê (8). Dos alimentos usualmente encontrados em saladas e ensopados, observa-se a citação majoritária do tomate e do alface (10), seguida de outras citações com menos de $50 \%$ dos questionários (chuchu, couve, vagem, cenoura entre outras leguminosas e folhas).

No entanto, ao serem questionados sobre o gosto por tais alimentos, os entrevistados mostraram discrepância nos resultados, pois o arroz (12), o feijão (11) e a carne vermelha (8) tiveram menos citações. 0 macarrão, o tomate (10) e 0 alface (8) são consumidos e fazem parte do gosto deles.

0 confronto entre gostar e consumir mostrou que, em relação à batata, ocorreu o contrário do que foi apresentado no parágrafo anterior, pois mais adolescentes relataram gostar deste alimento, embora não tenham informado que o consumam 
comumente: 12 gostam de purê, 11 gostam dela frita e 8 a preferem cozida. A omelete, que não havia sido citada no item dos alimentos comumente consumidos, destacou-se nos resultados sobre o gosto dos adolescentes, sendo citada por $50 \%$ deles. Outras citações tiveram presença inexpressiva, com menos de $30 \%$ dos questionários.

Sobre as frutas, as que fazem parte do hábito de consumo diário mostram dados satisfatórios em relação à variedade, uma vez que todos relataram comer maçã (14), e a maioria pera e manga (11), banana (10), melancia e abacaxi (8), mamão (7). No que tange ao gosto por estas frutas, observase que a maçã (12) teve menos citações, assim como a manga (9) e o abacaxi (7). A melancia (9) e a banana (11) apareceram com mais uma citação, e a uva (8) foi citada como sendo do gosto deles, embora não tenha aparecido na relação dos hábitos diários de consumo.

Nas perguntas sobre quais guloseimas e sanduíches os adolescentes têm por hábito ingerir, os resultados obtidos são considerados preocupantes, pois foram majoritárias as respostas em relação às balas e doces (13), salgadinhos (11), biscoitos recheados e salgados (11), e também o consumo de pizza (11). Os sanduíches que tiveram maior incidência de consumo foram o misto-quente (12) e o hambúrguer (9), seguidos de outras citações que não tiveram grande expressão nos dados totais, tais como: cheeseburguer (5), eggcheeseburger (3), "Big Mac" (4) e "Big Bob"(2).

Outra refeição diária analisada foi o desjejum. Os alimentos mais citados como comumente ingeridos foram: 0 pão (12) com margarina ou manteiga (9), presunto (8) e queijo branco (7), acompanhados de bebidas como achocolatados (13), leite e suco (9). Observa-se que, à luz dos dados desta pesquisa, o café não integra o hábito e nem o gosto do adolescente.

0 consumo de refrigerantes despertou atenção, pois 13 dos 14 adolescentes entrevistados declararam ter o hábito de ingerir este tipo de bebida. Com tal resultado, buscou-se identificar em quais ocasiões eles os consomem. Nove deles declararam ingeri-los às refeições, e 7 a qualquer hora do dia e quando lancham fora de casa. Menos expressivo foi o consumo de refrigerantes no recreio do colégio (1), o que pode se justificar pelo fato de não haver disponibilidade de sua venda, pois não há cantina neste colégio.

Quanto à periodicidade da ingesta de refrigerantes, 5 adolescentes os ingerem todos os dias; 4 , somente nos finais de semana; outros 4 , de três a quatro dias por semana. 0 adolescente que informou não ter o hábito de beber refrigerante afirmou só fazê-lo em situações festivas.

A questão sobre a ingestão de água gerou respostas muito variadas, o que mostrou a necessidade de intervenção que deve ser feita no intuito de estimular os adolescentes ao seu consumo e evidenciando a sua importância para os processos fisiológicos e a saúde. A maior citação foi a de ingestão de 6 copos por três adolescentes, seguidos de 5 copos por outros dois. Apenas um adolescente informou ingerir menos de 1 copo.
Um, dois, sete, oito e quinze copos de água diários tiveram uma citação cada. A referência adotada foi a de 1 copo com capacidade para $200 \mathrm{ml}$ (mililitros).

\section{Alimentação saudável: 0 que diz 0 adolescente sobre esta prática}

-Autoavaliação das práticas alimentares pelos adolescentes

$\mathrm{Na}$ análise dos depoimentos à luz dos hábitos alimentares informados pelos adolescentes, observa-se que há certo padrão de informação sobre o que seja uma alimentação dita saudável, que engloba sempre a citação de muita salada, verduras, legumes e frutas. A variedade e 0 equilibrio, em termos de quantidade, surgem nos depoimentos; no entanto, os dados numéricos sobre o consumo cotidiano dos alimentos pelos adolescentes informam que eles não seguem o padrão que consideram ser saudável.

"Ah, comer todos os alimentos não em muita quantidade e só." (M3-14)

"Comer de tudo um pouco" (F2-16)

"Eu acho que tem que ser uma alimentação bem variada, com verduras e carnes, mas não só isso. Sempre variando bastante." (F4-13)

"Comer vegetais e frutas e não exagerar muito em comer carnes, beber refrigerante essas coisas." (F5-14)

Quando solicitados a fazer autoavaliação sobre a qualidade de sua alimentação, os adolescentes não foram unânimes em considerar que praticam uma alimentação saudável, mostrando uma condição de crítica e autocrítica sobre seus próprios hábitos. Seis adolescentes manifestaram-se assumindo que a alimentação praticada não se alinha com 0 que se preconiza como saudável.

"Eu não acho não, porque eu nem como frutas direito e eu também não como verdura, e como muita coisa gordurosa, com óleo e também doces e balas. Eu não penso em mudar não, e minha mãe, apesar de não ser assim, também nem briga comigo" (M2-14)

"Não, porque eu como muita gordura, muito docee muito refrigerante e é raro eu comer alguma coisa que seja saudável"( F3-13)

Outros seis avaliaram os tipos de alimento e sua preparação, considerando que seus hábitos alimentares são saudáveis. 
"Sim, porque eu tento comer de tudo um pouco, variando bastante os alimentos, não como somente gorduras e somente verduras, como de tudo." (F413)

"Sim, pela variedade de coisas que eu como todos os dias" (M3-14)

Dois deles não conseguiram se posicionar de forma segura e avaliaram sua alimentação com indecisão na resposta: "mais ou menos" (F1-13, F9-14).

Um dos adolescentes demonstrou a preocupação com o peso, relacionando a dita boa alimentação com o ganho ponderal.

"Sim, porque eu tenho que comer bem para jogar bola, não engordar e ficar bem sempre." (M1-14)

- Comidas de preparação rápida como estilo de alimentação na adolescência

Todos os adolescentes entrevistados reconheceram a alimentação de preparação rápida, o típico para esta fase. Foram citados como alimentos e preparações que marcam a identidade adolescente os sanduíches, principalmente hambúrgueres, pizza, batata frita, além de guloseimas, como doces diversos. Os adolescentes fizeram questão de enfatizar que eram típicos os alimentos com mais gordura, citando principalmente as frituras. Apenas um entrevistado, apesar de ter a mesma opinião sobre esse tipo de alimentação, relatou não fazê-la.

"[...] como pizza, hambúrguer e batata frita, eu como isso" (M1-14)

"Sim eu acho até que adulto também come, mas a gente come muita batata frita, pizza... Ah, eu amo, então como muito." (F9-14)

"Sim, MacDonald's, hambúrguer essas coisas, mas eu não costumo comer." (F1-13)

Observa-se, nos depoimentos, inclusive, a alusão a tal tipo de alimentação como constituída por "besteiras", mostrando que os adolescentes, de um modo geral, têm conhecimento sobre os alimentos e preparações consideradas saudáveis, e outros cujo consumo deve ser moderado ou até evitado, na dependência da situação. Além disso, mostram a força da mídia na substituição do nome de uma forma de apresentação de alimentos (sanduíches) pelo nome de uma grande rede que os comercializa

"Eu acho que sim, as besteiras como refrigerante, MacDonald's que a gente sempre vai" (F6-14)
"Algumas besteiras como hambúrguer, batata frita, pizza e também frango, essas coisas." (F2-16)

"Sim, muito doce, salgadinhos essas besteiras." (F3-13)

Justificam a opção por este tipo de alimentação devido à falta de tempo que têm para se alimentar, relacionando-a à rapidez de seu preparo e, portanto, alternativa para quem não tem tempo para se alimentar como deveria.

"Sim, os doces, balas, até porque as coisas que nós comemos no recreio, com o tempo que temos, tem que ser uma comida mais rápida, então não somos muito obrigados a comer legumes." (F7-15)

A palavra "besteira" empregada por alguns adolescentes, exemplificada em algumas das unidades de registros citadas, classifica os alimentos em preparação rápida, de forma a desqualificá-los. Ainda assim, o consumo existe e parece sustentar certa identidade própria da idade. É como se aqueles que não consumissem este tipo de preparação estivessem alijados de um processo de adolescer.

- Relação alimentação e saúde: os nexos atribuídos pelos adolescentes

A relação entre alimentação e saúde foi estabelecida por 13 dos adolescentes entrevistados. A exceção ficou por conta de um deles, quando afirmou que alimentos considerados não saudáveis, se forem ingeridos de forma não cotidiana, não trarão grande impacto para a saúde.

"[Minha alimentação] Eu acho que está mais ou menos, porque se eu comer hambúrguer só algumas vezes e depois fizer uma alimentação saudável, só isso não vai fazer tão mal assim" (M1-14)

"Dependendo de como a pessoa se alimenta, ela melhora ou não sua saúde cada vez mais." (F2-16)

Logo, a alimentação guardou nexos importantes com a saúde na opinião da maioria dos adolescentes, inclusive com citações pontuais de determinadas enfermidades que estão diretamente ligadas ao estilo de vida. Também foi citado que a alimentação saudável só passa a ser habitual a partir do momento em que o indivíduo apresenta algum tipo de doença. Em sua maioria, os adolescentes acabaram por relacionar alimentação saudável com melhorias no estilo de vida e a prevenção de doenças. 
"A pessoa que come muita besteira acaba tendo problema do coração, essas doenças." (F6-14)

"Eu acho [que alimentação tem a ver com saúde], não sei, mas uma vez eu fiquei sem me alimentar e desmaiei na escola, aí eu comecei a me cuidar mais e comer melhor." (F1-13)

"Dependendo de como a pessoa se alimenta ela melhora ou não sua saúde cada vez mais." (F216)

"Os alimentos que nós comemos tem a ver com a nossa saúde, alguns combatem doenças e os que comem direito vivem até melhor também." (F7-15)

"Com os alimentos adequados as pessoas passam a prevenir diversas doenças." (F8-14)

"Às vezes, se eu comer muita coisa boa como vitamina é bom, mas tem gente que fica doente, aí sim passa a pensar em comer coisa boa e vitamina. Mas é bom sempre você ter uma alimentação boa porque então não precisa ficar doente." (F9-14)

Com isso se observou que as mudanças de comportamento, no que tange à alimentação, podem ser realizadas a partir do momento que o adolescente compreender realmente os enfrentamentos que irão perdurar ao longo da vida em consequência de sua alimentação.

\section{DISCUSSÃO}

Lidar com a adolescência é abrir novas oportunidades de orientação nutricional e educação em saúde, já que o consumo alimentar adotado nessa fase da vida tem sérias implicações na fase adulta, aumentando ainda mais a dificuldade de modificá-los. A dinâmica familiar assume papel considerável na mudança dessas práticas. ${ }^{4}$ Pode-se inferir também que, para 0 adolescente modificar seus hábitos alimentares, é fundamental primeiramente reconhecê-los através de autoavaliação e autocrítica.

Os resultados numéricos da pesquisa demonstraram um leque de informações sobre alimentação saudável baseadas na variedade, equilíbrio e concentração no consumo de leguminosas e frutas. Por outro lado, foi frequente o consumo de alimentos mais gordurosos e guloseimas comumente consumidas na fase da adolescência que acabam se caracterizando como comidas típicas de preparação rápida acompanhadas de alta ingestão de refrigerantes. $A$ base da alimentação não deve se sustentar nestes tipos de preparações, pois este tipo de consumo, rico em gordura poli-insaturada e em carboidratos simples, associado à diminuição, de níveis populacionais, de gastos energéticos através de atividades físicas, são consistentes com a importância crescente de doenças crônicas não transmissíveis, como o caso da diabetes mellitus e problemas cardiovasculares, no perfil de morbimortalidade e com o aumento contínuo da prevalência de obesidade no País. ${ }^{13}$

Uma questão favorável do estudo foi o consumo de frutas e verduras, os quais foram relatados por todos os entrevistados, mesmo que em pouca quantidade e variedade, o que de se distancia de resultados e conclusões de estudos feitos com adolescentes com a mesma faixa etária desta pesquisa. ${ }^{14}$ Houve, também, incidência nos relatos sobre o consumo de guloseimas como balas e doces, havendo prevalência total de ao menos um tipo de citação para cada adolescente.

0 café da manhã também foi avaliado, em frequência e conteúdo, sendo encontrada boa variedade de alimentos consumidos nessa refeição, o que é satisfatório já que esta refeição é de extrema importância para a condução das atividades diárias praticadas pelos adolescentes que necessitam de grande demanda energética. Ademais, levando-se em conta que a pesquisa foi feita no primeiro turno do Colégio, o café da manhã é crucial para que os adolescentes possam ter bom rendimento nas atividades escolares e sucesso na aprendizagem, já que a baixa glicemia traz déficit de atenção e sonolência.

0 número de refeições que os adolescentes realizam dá-se muito por influência das tradições, do nível socioeconômico e educacional da família, da influência dos colegas e até da mídia. ${ }^{15}$ Nesse sentido, os resultados desta pesquisa foram favoráveis, uma vez que a minoria dos adolescentes ficava sem realizar algum tipo de refeição diária mantendo grandes intervalos, pois as implicações de um fracionamento inadequado da dieta e de um descontrole de horários para se alimentar podem incluir problemas decorrentes do jejum prolongado, como maior propensão à gastrite ou realização de refeições excessivamente volumosas para "compensar" o jejum. ${ }^{15}$

Outro questionamento importante foi em relação à autoavaliação sobre as práticas alimentares dos adolescentes e se eles relacionavam a saúde com uma alimentação saudável. Sobre isso, os resultados apontaram que, apesar de os adolescentes saberem que não se alimentavam bem, eles não faziam nenhuma mudança, e que, apesar de a maioria considerar ter uma prática inadequada, ainda assim apontava que a alimentação é a base para a boa saúde. Isso mostra que os adolescentes possuem visão crítica sobre a alimentação, mas ainda não conseguem implementar os hábitos de uma alimentação saudável em prol de sua própria saúde. Não conseguem, talvez, por influência do meio em que eles vivem, de uma cultura de consumo de lanches rápidos e comidas práticas, e estímulos à criação de uma certa identidade da infância e do adolescer ligados a redes de lanchonetes. Essas observações revelam o quão importante é o tema da alimentação na infância e na adolescência. 
Um fator de risco que se encontra extremamente interligado com hábitos de alimentação que não sejam saudáveis é a predominância de doenças cardiovasculares. Adolescentes que apresentam níveis de pressão arterial aumentados são aqueles mais propensos a desenvolverem estes tipos de doenças, ${ }^{16}$ e sabe-se que alimentos pré-preparados, com embutidos principalmente, têm grande teor de sódio, concorrendo para a retenção hídrica e elevação da pressão arterial.

Isso se evidencia nas unidades de registro analisadas, onde se destacam os relatos de consumo destes tipos de alimentos, podendo trazer consequências futuras, na dependência das disposições genéticas e do cultivo de tais hábitos na idade adulta. Daí se ressalta que o consumo quantiqualitativo de alimentos aliado a atividades físicas integram o conjunto de ações de programas de saúde pública na prevenção de enfermidades, mormente nesta fase da vida - a adolescência.

Dessa maneira, pela análise dos dados e discussão dos resultados, pode-se identificar que intervenções de sucesso em prol de uma alimentação saudável tornam-se um desafio para o campo da saúde pública, em especial, na área da saúde do adolescente. Considerando-se o locus da pesquisa, há que se considerar em tais intervenções, as características do modo de vida urbano que orientam o comportamento alimentar ${ }^{17}$ dos adolescentes.

\section{CONSIDERAÇÕES FINAIS}

À luz dos resultados pode-se destacar que foram atendidos os objetivos da pesquisa, uma vez que, ao se explorar os saberes dos adolescentes sobre o que seja uma alimentação saudável, evidenciou-se os alimentos mais e menos consumidos e a relação do consumo com o gosto por eles, o que leva a concluir que quando não se desenvolve ainda na infância o paladar para determinados sabores e consistências, torna-se difícil, mais adiante, incorporar certos tipos de alimentos no cardápio diário.

Apesar de os adolescentes terem a informação sobre o que seriam hábitos saudáveis de alimentação, a maioria declarou não os seguir, o que mostra que a informação não é a única dimensão de uma prática, mas apenas parte de uma grande engrenagem influenciada pelo contexto sociocultural de formação de identidades.

Em relação à metodologia utilizada, esta teve grande aplicabilidade nesta investigação, uma vez que possibilitou entrosamento entre a pesquisadora e o adolescente entrevistado, possibilitando a este expressar suas opiniões e atitudes, além de esclarecer suas eventuais dúvidas sobre o assunto, oportunizando a realização de cuidados de enfermagem através da educação em saude.

Ainda sobre o método aplicado, este estudo contribuiu para que houvesse, por parte dos adolescentes, a compreensão das próprias práticas alimentares, através da reflexão e autocrítica sobre seus hábitos, o que contribuiu para que pudessem repensar as suas práticas e buscassem estratégias para mudanças em seus hábitos cotidianos.

Vale ressaltar que a educação em saúde deve ser entendida como um instrumento de ação para o enfermeiro atuar junto ao adolescente, promovendo sua saúde através da valorização de seus saberes, o que favorece a construção de uma proposta de ação terapêutica em que os clientes estejam mais ativos, e não passivos nos cuidados. Desta forma, esperase que se tenham melhores resultados na atenção a estes sujeitos do cuidado. Assim, há que se considerar a cultura alimentar dos adolescentes e jovens em ações de educação em saúde, promovendo a crítica-reflexiva dos sujeitos com vistas à promoção da saúde e qualidade de vida.

Considerando o critério de expansibilidade que norteia a pesquisa convergente-assistencial, esta investigação, de cunho exploratório, aponta possibilidades de outros temas para pesquisas futuras, com ampliação do número de sujeitos e abrangência de faixa etária, para que se possa ter mais segurança nas comparações com outros estudos realizados sobre a alimentação na adolescência, pois o quantitativo de sujeitos e a sua realização em somente um colégio mostram-se como limitantes para se ampliarem as discussões e a proposta de estratégias de intervenção no campo educativo em saude.

Adianta-se que uma possibilidade vislumbrada para continuidade deste projeto é a realização de pesquisa que vise mostrar a existência ou não de transição de hábitos de alimentação na adolescência, pois isto pode contribuir em muito com as ações do campo da saúde pública, no que pese à saúde do adolescente.

\section{REFERÊNCIAS}

1. Albano RD, Souza SB. Ingestão de energia e nutrientes por adolescentes de uma escola pública. J Pediatr. 2001; 77; 512-16.

2. Canesqui AM, Garcia RWD. Uma introdução à reflexão sobre a abordagem sociocultural da alimentação. Antropologia e nutrição: um diálogo possível. Rio de Janeiro: Ed Fiocruz; 20054 p. 9-19.

3. Ministério da Saúde(BR)Secretaria de Atenção à Saúde. Departamento de atenção básica. Política Nacional de Alimentação e Nutrição, 2 aed. Brasilia (DF); 2003.

4. Rodriguez EM, Boog MC. Problematização como estratégia de educação nutricional com adolescentes obesos. Cad Saude Publica. 2006 maio; 22(5); 923-931, maio de

5. Veiga GV, Cunha AS, Sichieri R. Trends in overweight among adolescents living in the poorest and riches regions of Brazil. Am J Public Health. 2004; 94(9): 1544-48. 
6. Ressel LB, Hoffman IC, Sehnem GD, Landerdahl MC, Junges CF. Representações culturais de saúde, doença e vulnerabilidade sob a ótica de mulheres adolescentes. Esc. Anna Nery. 2009 jul-set: 552-57.

7. Bertin RL, Karkle ENL, Ulbrich AZ, Neto AS, Bozza R, Araujo IQ, Campos W. Estado Nutricional e consumo alimentar de adolescentes da rede pública de ensino da cidade de São Mateus do Sul, Paraná, Brasil. Rev Bras Saude Matern Infant. 2008 out/dez; 8 (4): 435 - 43.

8. Toral N, Slater B, Silva MV. Consumo alimentar e excesso de peso de adolescentes de Piracicaba, São Paulo. Rev Nutr, Campinas, 2007 set./ out; 20 (5): 449-59.

9. Ministério da Saúde (BR). Secretaria de Programas e Projetos. Divisão de Saúde Materno-Infantil. PROSAD - Bases Programáticas. Brasília (DF); 1989.

10. Vilela JEM, Lamounier JA, Filho MAD, Neto JRB, Horta GM. Transtornos alimentares em escolares. J Pediatr. 2004; 80(1).

11. Trentini M, Paim L. Pesquisa convergente assistencial: um desenho que une o fazer e o pensar na prática assistencial em saúde-enfermagem. $2^{\mathrm{a}}$ ed. Florianópolis: Insular; 2004.

12. Chiara VL, Sichieri R, Carvalho TSF. Teores de ácidos graxos trans de alguns alimentos consumidos no Rio de Janeiro. Rev Nutr. 2003 abr/ jun;16(2):227-33.

13. Neutzling MB, Araújo CLP, Vieira MFA, Hallal PC, Menezes ANB Freqüência de consumo de dietas ricas em gordura e pobres em fibra entre adolescentes. Rev Saude Publica. 2007; 41(3): 336-42.

14. Vieira VCR, Priore SE, Ribeiro SMR, Franceschini SCC, Alterações no padrão alimentar de adolescentes com adequação pôndero-estatural e elevado percentual de gordura corporal. Rev Bras Saude Matern Infant $2005 \mathrm{jan} / \mathrm{mar} ;$ 5(1): 93-102.

15. Campana EMG, Brandão AA, Pozzan R, França MR, Fonseca FL, Pizzi OL, Magalhães MEC, Freitas EV, Brandão AP. Pressão arterial em jovens como marcador de risco cardiovascular: estudo do Rio de Janeiro. Arq Bras Cardiol. 2009; 93(6) : 657-65.

16. Marinho MCS, Hamann EM, Lima ANCCF. Práticas e mudanças no comportamento alimentar na população de Brasilia, Distrito Federal, Brasil. Rev Bras Saude Matern Infant. 2007 jul /set; 7 (3): 251-61.

17. Ferreira, MA, Alvim NAT, Teixeira MLO, Veloso RC. Saberes de adolescentes: estilo de vida e cuidado à saúde. Texto\&Contexto Enferm. 2007 abr-jun; 16(2): 217-24 